\title{
Active galactic nuclei and their host galaxies
}

\author{
W. H. Bian ${ }^{1,2} \dagger$, Y. H. Zhao ${ }^{2}$ \\ and L. P. Cheng ${ }^{2}$ \\ ${ }^{1}$ Department of Physics, Nanjing Normal University, Nanjing 210097, China \\ email: whbian@njnu.edu.cn \\ ${ }^{2}$ National Astronomical Observatories, Chinese Academy of Sciences, Beijing 100012, China \\ email:yzhao@lamost.bao.ac.cn
}

\begin{abstract}
In recent years there has been rapid progress in the research of Active Galactic Nuclei (AGNs), such as central super-massive black holes (SBH), accretion processes, and their host galaxies. Using data from ROSAT point sources, we found that the soft X-ray spectra of narrow-line Seyfert 1 galaxies (NLS1s) and Blazars become harder during X-ray flux increases, while the spectra of broad-line AGNs (BL AGNs) become softer. From ASCA data, we found that NLS1s and BL AGNs follow the same $\sigma^{2}-M_{M B H}$ relation, where $\sigma^{2}$ is the excess variance in the hard X-ray band. With the accretion efficiency of AGNs, we found that most of the radioloud quasars possibly possess a Kerr SBH, while the Seyfert 1 galaxies and radio-quiet quasars possess Schwarzschild SBH. We also studied the relation between the central black holes and their host galaxies. It was found that NLS1s appear to have genuinely lower MBH/bulge mass ratios. And the width of the [O III] line is not a good tracer of host velocity dispersion. NLS1s play a special role in our understanding of the evolution of galaxies and their central SBHs.
\end{abstract}

\section{Introduction}

As we know, Active Galactic Nuclei (AGNs) consist of central super-massive black holes (SBHs) harboured in their host galaxies, with a jet and emission line regions. Based on the unified model of AGNs, the wide variety of AGN phenomena we see are due to a combination of real differences in a small number of physical parameters (e.g. luminosity, mass) coupled with apparent differences which are due to observer-dependent parameters (e.g. orientation) (Urry \& Padovani 1995). With the reverberation mapping technique, SBH masses can be reliably determined in about three dozen AGNs (Kaspi et al. 2000). The advantage of knowing SBH masses is that it gives an additional constraint on accretion theory, which depends only on the accretion rate and the inclination. At the same time we can also calculate the Eddington ratio of the bolometric luminosity to the Eddington luminosity, which can be used as a measure of the strength of the accretion process and is related to "eigenvector 1", the fundamental parameter of the central engine (Boroson \& Green 1992). From the unified galaxy evolution model, in the early evolutionary stages, the nuclei of galaxies with strong accretion appear as AGNs. Evidence shows that there is a strong connection between AGN and their host galaxies. There are two outstanding correlations. One is the SBH-to-bulge mass ratio. The other is the relation between $\mathrm{SBH}$ mass and the host velocity dispersion ( $M_{S B H}-\sigma$ relation). Here we review our recent work on AGNs and their host galaxies.

$\dagger$ Present address: 120, Ninghai Road, Nanjing, Jiangsu Province, P. R. China. 


\section{SBH masses and the Eddington ratio}

It is essential to derive reliable SBH masses in AGNs. Up to now, there are a number of methods which have been used to estimate SBH masses (Bian 2004, and the references therein): (i) the virial mass derived from the $\mathrm{H} \beta$ FWHM and the sizes of broad line regions (BLRs) from the reverberation mapping technique, or the empirical size - luminosity formula; (ii) soft X-ray variability; (iii) the relation between the mass and the bulge stellar velocity dispersion $\left(M_{S B H}-\sigma\right.$ relation $)$ or the bulge luminosity $\left(M_{S B H}-L_{b u l g e}\right.$ relation). With SBH masses, we can obtain the Eddington ratio of the bolometric luminosity $L_{b o l}$ to the Eddington luminosity $L_{E d d}$. $L_{b o l}$ is usually calculated as $L_{b o l}=9 L_{\lambda}(5100 \AA)$, where $L_{\lambda}(5100 \AA)$ is the monochromatic luminosity at $5100 \AA$.

\section{X-ray variability}

$\mathrm{X}$-ray variability is a common property in AGNs. Its origin is still a question open to debate. The rapid variability is usually thought to come from the innermost region of the compact objects, and is helpful in getting information about the central objects, such as mass, accretion rate, geometry, and radiation mechanisms. We assembled a sample of broad-line AGNs and NLS1s observed by ASCA, the excess variances of which have been determined (Bian \& Zhao 2003a). We found that NLS1s and BL AGNs follow the same $\sigma^{2}-M_{S B H}$ relation, where $\sigma^{2}$ is the excess variance in hard X-ray band (see Fig. 1). And that there is almost no correlation between the X-ray variability and the Eddington ratio, which suggests that rapid variability and narrow lines in NLS1s are mainly due to small central black holes in NLS1s, and not to differences in the circum-nuclear gas around NLS1s. At the same time, a strong correlation was found between the hard X-ray photon index and the Eddington ratio. Using ROSAT point data, we also found that the soft X-ray spectra of NLS1s and Blazars became harder during X-ray flux increases, while the spectra of broad-line Seyfert 1 galaxies became softer (Cheng, Zhao \& Wei, $2002 \mathrm{a}, \mathrm{b})$.

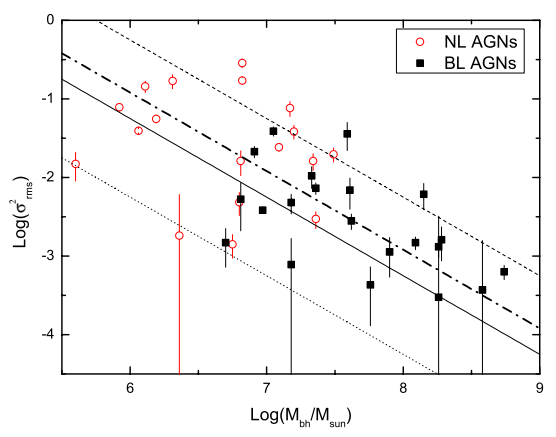

Figure 1. Excess variance in the hard X-ray band versus the central black hole mass.

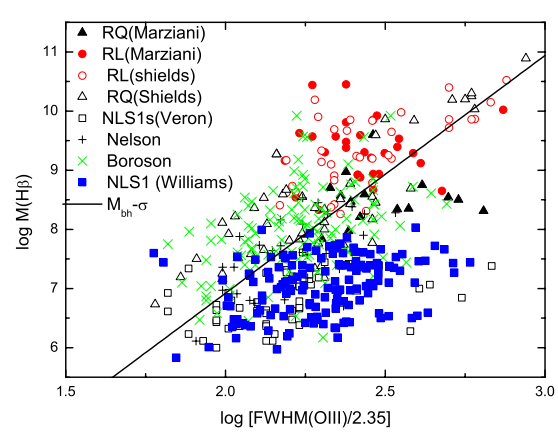

Figure 2. Black hole masses derived from the $\mathrm{H} \beta$ line width versus the width of the [O III] line for AGNs.

\section{Accretion efficiency and SBH rotation}

Knowing the SBH masses, we can obtain accretion rates from the standard accretion in AGNs. Combining the accretion rates with the bolometric luminosity, the accretion 
efficiency can be calculated (Bian \& Zhao 2003c). We found that most Seyfert 1 galaxies and radio-quiet quasars have lower accretion efficiencies, while most radio-loud quasars possess higher accretion efficiencies. For a non-rotational black hole, the maximum accretion efficiency converting the accretion mass to energy is $5.7 \%$, while for a maximally rotating one the accretion efficiency is $32.4 \%$. Therefore, our finding further implies that most of the radio-loud quasars possess Kerr black holes, while Seyfert 1 galaxies and radio-quiet quasars may not possess Kerr black holes. This provided new clues to the dichotomy of radio-quiet and radio-loud AGNs.

\section{The host galaxies of AGNs}

Evidence shows that the evolution of black holes and that of their host galaxies appear to be closely coupled. There is a strong correlation between SBH mass and the host velocity dispersion $\left(M_{S B H}-\sigma\right.$ relation). The mass ratio of SBH mass to its bulge is also a constant, about $10^{-3} \cdot M_{S B H}$ is estimated from the $\mathrm{H} \beta$ line width and $\sigma$ is directly estimated from the width of the [O III] line. We found that radio-quiet AGNs follow the $M_{S B H}-\sigma$ relationship, while radio-loud AGNs and NLS1s deviate from this relationship (Bian \& Zhao 2004a) (see Fig. 2). With the bulge luminosity, the MBH/bulge mass ratio of NLS1s is genuinely lower than broad-line AGNs, which is consistent with the scenario that NLS1s are active galaxies in an early stage of evolution (Bian \& Zhao 2003b). Our results depended on the SBH masses estimate in NLs1s. New methods are needed to derive accurate SBH masses in NLS1s (Bian \& Zhao 2004b).

\section{Acknowledgements}

W.-H. Bian is supported by the NSFC (No. 10403005; No. 10473005; No. 10273007) and the NSF of Jiangsu Provincial Education Department (No. 03KJB160060).

\section{References}

Bian, W. H., 2004, PhD thesis, National Astronomical Observatories, Chinese Academy of Sciences, China

Bian, W. H., Zhao, Y. H., 2003a, MNRAS, 343, 164

Bian, W. H., Zhao, Y. H., 2003b, PASJ, 55, 143

Bian, W. H., Zhao, Y. H., 2003c, PASJ, 55, 599

Bian, W. H., Zhao, Y. H., 2004a, MNRAS, 347, 607

Bian, W. H., Zhao, Y. H., 2004b, MNRAS, 352, 823

Boroson, T. A., Green, R. F., 1992, ApJS, 80, 109

Cheng, L. P., Zhao, Y. H., Wei, J. Y., 2002a, ChJAA, 2, 117

Cheng, L. P., Zhao, Y. H., Wei, J. Y., 2002a, ChJAA, 2, 207

Kaspi, S., Smith, P. S., Netzer, H., Maoz, D., Jannuzi, B. T., Giveon, U., 2000, ApJ, 533, 631

Urry, C. M., Padovani, P., 1995, PASP, 107, 803 